\title{
PHYSIOLOGICAL POTENTIAL OF CAULIFLOWER SEEDS
}

\author{
Ana Lúcia Pereira Kikuti ${ }^{1}$; Julio Marcos-Filho ${ }^{2 *}$ \\ ${ }_{2}^{1} U S P / E S A L Q$ - Programa de Pós-Graduação em Fitotecnia. \\ ${ }^{2}$ USP/ESALQ - Depto. de Produção Vegetal, C.P. 09 - 13418-900 - Piracicaba, SP - Brasil. \\ *Corresponding author<jmarcos@esalq.usp.br>
}

\begin{abstract}
The assessment of seed physiological performance is essential to provide consistent information to compose quality control programs conducted by seed companies and allow prompt decisions to reduce post-harvest and commercialization problems. This research was carried to evaluate the efficiency of different procedures to determine cauliflower seed vigor. Six lots of the cultivar Sharon were initially characterized by germination and seedling emergence, and subsequently subjected to traditional and saturated salt accelerated aging, controlled deterioration, electrical conductivity, and potassium leachate tests, in two evaluation times. The traditional accelerated aging $\left(41^{\circ} \mathrm{C} / 48 \mathrm{~h}\right)$, the saturated salt procedure $\left(45^{\circ} \mathrm{C} / 72 \mathrm{~h}\right)$, the controlled deterioration (moisture content adjusted to $20 \%$ or $22 \%$ and aging at $45^{\circ} \mathrm{C} / 24 \mathrm{~h}$ ), the electrical conductivity $(50$ seeds $/ 75 \mathrm{~mL}$ water and evaluation after $4 \mathrm{~h}$ ) and the potassium leakage $(25 \mathrm{seeds} / 50 \mathrm{~mL}$ of water and evaluation after $30 \mathrm{~min}$ ) laboratory tests are sensitive to detect differences in the physiological potential of cauliflower seeds. However, those results did not relate to the percentage seedling emergence in greenhouse controlled conditions, as a consequence of the favorable conditions for emergence. However, the differences identified in the tests may be related to the ability of seed lots to perform in less favorable environments.
\end{abstract}

Key words: Brassica oleracea L. var. botrytis, seed analysis, vigor, quality control

\section{POTENCIAL FISIOLÓGICO DE SEMENTES DE COUVE-FLOR}

RESUMO: A eficiência da determinação do potencial fisiológico de lotes de sementes é essencial para a obtenção de informações consistentes, permitindo a composição de programas de controle de qualidade de sementes e a obtenção de informações consistentes para a redução de problemas póscolheita e durante a comercialização. O objetivo desta pesquisa foi comparar a eficiência de diferentes procedimentos para a determinação do vigor de sementes de couve-flor. Para tanto, foram utilizados seis lotes do cultivar Sharon, caracterizados inicialmente pelos testes de germinação e de emergência de plântulas. Em seguida, conduziram-se testes de envelhecimento acelerado tradicional e com solução saturada de cloreto de sódio, deterioração controlada, condutividade elétrica e lixiviação de potássio, em duas épocas de avaliação. Os testes de envelhecimento acelerado tradicional, a $41^{\circ} \mathrm{C} / 48 \mathrm{~h}$, e com solução saturada de cloreto de sódio, a $45^{\circ} \mathrm{C} / 72 \mathrm{~h}$, de deterioração controlada (ajuste do grau de umidade para $20 \%$ ou $22 \%, 45^{\circ} \mathrm{C} / 24 \mathrm{~h}$ ), de condutividade elétrica (combinação 50 sementes $/ 75 \mathrm{~mL}$ de água/4 h) e de lixiviação de potássio (25sementes $/ 50 \mathrm{~mL}$ de água/30 min) são suficientemente sensíveis para detectar diferenças no potencial fisiológico de sementes de couve-flor. No entanto, essas informações não se refletiram na percentagem de emergência de plântulas em ambiente favorável, mas podem ser úteis para identificar lotes com maior probabilidade de apresentar desempenho adequado quando ocorrem desvios das condições mais adequadas de ambiente.

Palavras-chave: Brassica oleracea L. var. botrytis, análise de sementes, vigor, controle de qualidade

\section{INTRODUCTION}

The accurate identification of seed physiological potential is essential to select procedures to promote an adequate plant stand establishment and uniform seedling growth, particularly for species such as cauliflower for which commercial cropping operations involve transplants and other high technology practices. Although seed lots may have similar high germination values they can differ in the extent of deterioration and so exhibit differences in their ability to establish vigorous seedlings. As a consequence, various procedures have been developed for seed vigor determination of cultivated species.

Vegetable seed vigor may be assessed by traditional and saturated salt accelerated aging (TeKrony, 1995; Jianhua \& McDonald, 1997; Panobianco \& Marcos-Filho, 2001a; Fessel et al., 2005), and controlled deterioration tests (Powell \& Matthews, 1981; Torres \& Marcos-Filho, 2005; Basak et al., 2006). However, additional studies are still required for most vegetable species. 
In addition, rapid tests such as the electrical conductivity and potassium leakage have also been successfully employed to determine seed vigor of different species. It has been shown that the imbibition period for the electrical conductivity test can be reduced from 24 h to 4-8 hours (Martins et al., 2002, with broccoli; Oliveira \& Novembre, 2005, with pepper), the samples from 50 to 25 seeds and the water volume from $75 \mathrm{~mL}$ to $50 \mathrm{~mL}$ or $25 \mathrm{~mL}$ (Oliveira \& Novembre, 2005). In addition, results of the potassium leachate test which is based on a principle similar to the electrical conductivity are obtained within a considerably shorter period of time, e.g., after 30 to 90 minutes of imbibition (Custódio \& Marcos-Filho, 1997; Dias et al., 1998), but most of research has been performed on grain crops. These tests are usually conducted in seed quality control programs, but may still be opportunities to improve some aspects of the methodology from the perspective of standardization, accuracy, objectiveness, reduction in testing time, reproducibility within and among seed laboratories and reliability of results (Marcos-Filho et al., 2006).

Based on the importance of the vigor tests for vegetable seeds and the relatively few studies on cauliflower, the main objective of this research was to verify the efficiency of procedures to perform the accelerated aging, controlled deterioration, electrical conductivity and potassium leaching tests to identify vigor differences among cauliflower seed lots of high germinability. The vigor tests were chosen according to their rapidity, consistency and objectivity, including procedures based on the integrity of cell membranes and on tolerance to stress.

\section{MATERIAL AND METHODS}

This research was carried out in Piracicaba, state of São Paulo, Brazil $\left(22^{\circ} 42^{\prime} \mathrm{S}, 47^{\circ} 48^{\prime} \mathrm{W}\right)$ utilizing six lots of cauliflower seeds, cultivar Sharon. During the experimental period (ten months), seeds were maintained in paper bags under controlled conditions $\left(20^{\circ} \mathrm{C}\right.$ and $50 \%$ relative humidity). Tests were performed to evaluate seed moisture content and physiological potential at two evaluation times: June/August 2003 and February/April 2004. Seed storage conditions were considered favorable to maintain an adequate seed moisture content and preserve the physiological potential, as usual for most vegetable seeds.

Seed moisture content: evaluations were made using an oven at $105 \pm 3{ }^{\circ} \mathrm{C} / 24 \mathrm{~h}$ (Brasil, 1992). Results were expressed as mean percentage for each lot (fresh wet basis).

Germination: four replicates of 50 seeds from each lot were distributed in plastic boxes $(11.0 \times 11.0 \times 3.5$ $\mathrm{cm}$ ) on two sheets of blotter paper moistened with water equivalent to 2.5 times the paper weight and left for germination at $20-30^{\circ} \mathrm{C}$. Seedling evaluations were performed five and 10 days after sowing, according to the criteria established in the Brazilian Rules for Seed Testing (Brasil, 1992). Results were expressed as mean percentage of normal seedlings.

Seedling emergence: four replicates of 50 seeds were individually planted in multicell polystyrene trays containing a commercial growing mix. The trays were placed in a greenhouse and subjected to intermittent micro-sprinkler irrigation. Evaluations were performed 14 days after sowing; seedlings at least $1.0 \mathrm{~cm}$ in height were recorded and results were expressed as mean percentage of normal seedlings per lot.

Accelerated aging (traditional procedure): was performed in a "water jacketed" incubator with samples of approximately $4 \mathrm{~g}$ of seeds per lot, distributed as single layer on a wire mesh screen suspended over 40 $\mathrm{mL}$ of water inside a plastic accelerated aging box adapted as an individual compartment. The lid-covered boxes were maintained at $41^{\circ} \mathrm{C}$ and $45^{\circ} \mathrm{C}$ for $48 \mathrm{~h}$ and $72 \mathrm{~h}$, respectively; after these periods, four replicates of 50 seeds were tested for germination as described previously. Evaluation was performed five days after sowing and results were expressed as mean percentage of normal seedlings. Seed moisture content was also determined before and after the accelerated aging periods to evaluate the accuracy of the aging results.

Saturated salt accelerated aging: the same procedure described for the traditional test was used, except that $40 \mathrm{~mL}$ of a saturated $\mathrm{NaCl}$ solution substituted the water, thus providing a $76 \%$ relative humidity environment (Jianhua \& McDonald, 1997).

Controlled deterioration: seed moisture content of each lot was adjusted to $20 \%, 22 \%$, and $24 \%$ by the humid atmosphere method (Rossetto et al., 1995); each seed sample was then sealed in an aluminum foil packet and held in a refrigerator for five days at $10^{\circ} \mathrm{C}$ to achieve uniform water distribution. Following this period, seeds still in the foil packet were placed in a water bath at $45^{\circ} \mathrm{C}$ for $24 \mathrm{~h}$ (Powell, 1995). The germination after controlled deterioration was conducted as described above.

Electrical conductivity: the effects of 4,12 and $24 \mathrm{~h}$ imbibition periods in combination with seed number/ water volume $(25 / 25 \mathrm{~mL}, 25 / 50 \mathrm{~mL}, 50 / 50 \mathrm{~mL}$, and $50 /$ $75 \mathrm{~mL}$ ) were evaluated. The test was conducted with four subsamples per lot, weighed with precision of $0.0001 \mathrm{~g}$, placed into disposable plastic cups containing the respective volumes of distilled water and held 
in a germinator at $25^{\circ} \mathrm{C}$ for 4,12 or $24 \mathrm{~h}$. Following these periods, the electrical conductivity of leachates was determined with a DIGIMED DM-31 conductivity meter and the mean values were expressed as $\mu \mathrm{S}$ $\mathrm{cm}^{-1} \mathrm{~g}^{-1}$ seed.

Potassium leachate: different combinations of seed number and water volumes $(25 / 25 \mathrm{~mL}, 25 / 50 \mathrm{~mL}, 50 /$ $25 \mathrm{~mL}, 50 / 50 \mathrm{~mL}$ ) were evaluated. Four seed replicates were weighed with precision of $0.0001 \mathrm{~g}$ and placed into plastic cups containing the mentioned volumes of distilled water and kept in germinator at $25^{\circ} \mathrm{C}$.

The potassium leachate readings were performed after 30 and 120 minutes imbibition, using a DIGIMED NK-2004 flame photometer, adjusted to a $50 \mathrm{ppm}$ potassium pattern and reading 50. Potassium leakage was calculated as described by Panobianco \& Marcos-Filho (2001b). The results were expressed as ppm $\mathrm{K}^{+} \mathrm{g}^{-1}$ seed.

The statistical analysis was carried out separately for each test and evaluation time, according to a completely randomized design, to evaluate the differences among seed lots. Mean values were compared by the Tukey test $(p \leq 0.05)$.

\section{RESULTS}

The initial seed moisture content ranged from $7.5 \%$ to $7.8 \%$ at the first evaluation time, in 2003 , and from $8.1 \%$ to $8.4 \%$ at the second time, in 2004 , thus indicating that this parameter did not influence seed performance during germination and vigor testing.
Germination of six seed lots was high and similar, from $97 \%$ to $100 \%$, in the first evaluation time and from $80 \%$ to $93 \%$ in the second (Table 1). There were no differences at the first testing date, but at the second date the germination of lot 4 was lower than lots 3 and 5. No differences between lots were detected in the seedling emergence test at both evaluation times. However, at the second evaluation both germination and emergence were lower.

The traditional accelerated aging test and all procedures used for controlled deterioration indicated the lowest performance of seed lot 4 in both evaluation times (Table 2). However, in the traditional accelerated aging at $41^{\circ} \mathrm{C} / 48 \mathrm{~h}$ results were more consistent between evaluation times than the exposure for $72 \mathrm{~h}$. In the controlled deterioration test, when seeds were hydrated to reach $24 \%$ moisture content, the mean values tended to cluster. For this reason, the adjustments to $20 \%$ or $22 \%$ prior to the water bath were considered more satisfactory. In addition, these tests confirmed the highest physiological potential of seed lot 5 , in some cases not different from lot 3 .

The traditional accelerated aging test at $45^{\circ} \mathrm{C}$ for $48 \mathrm{~h}$ caused drastic effects, and after $72 \mathrm{~h}$ aging period no germination occurred (Table 2). Considering all temperature - exposure periods, only the combination $45^{\circ} \mathrm{C} / 72 \mathrm{~h}$ resulted in variations in seed water content over the tolerable limit reported by MarcosFilho (1999).

The effects of saturated salt accelerated aging were less drastic in relation to the traditional proce-

Table 1 - Moisture content, germination and seedling emergence of six cauliflower seed lots, cv. Sharon, in two evaluation times.

\begin{tabular}{|c|c|c|c|c|}
\hline Evaluation & Lots & Germination & Seedling emergence & Moisture content \\
\hline & & (n) & - $\%$ - & - \\
\hline & 1 & $99 \mathrm{a}$ & $100 \mathrm{a}$ & 7.7 \\
\hline & 2 & $98 \mathrm{a}$ & $98 \mathrm{a}$ & 7.8 \\
\hline $1^{\mathrm{st}}$ & 3 & 99 a & $100 \mathrm{a}$ & 7.7 \\
\hline \multirow[t]{3}{*}{ (2003) } & 4 & $100 \mathrm{a}$ & 99 a & 7.6 \\
\hline & 5 & 97 a & $100 \mathrm{a}$ & 7.8 \\
\hline & 6 & $97 \mathrm{a}$ & 99 a & 7.5 \\
\hline \multirow[t]{3}{*}{$\mathrm{CV}(\%)$} & & 6.2 & 4.8 & \\
\hline & 1 & $90 \mathrm{ab}$ & $86 \mathrm{a}$ & 8.4 \\
\hline & 2 & $86 a b$ & $77 \mathrm{a}$ & 8.1 \\
\hline $2^{\text {nd }}$ & 3 & $93 \mathrm{a}$ & $83 \mathrm{a}$ & 8.3 \\
\hline \multirow[t]{3}{*}{ (2004) } & 4 & $80 \mathrm{~b}$ & $84 \mathrm{a}$ & 8.4 \\
\hline & 5 & $93 \mathrm{a}$ & $84 \mathrm{a}$ & 8.2 \\
\hline & 6 & $83 \mathrm{ab}$ & $86 \mathrm{a}$ & 8.1 \\
\hline $\mathrm{CV}(\%)$ & & 6.4 & 5.1 & \\
\hline
\end{tabular}

Lower case letters: lot comparison within each phase (Tukey test, $p \leq 0.05$ ).

Sci. Agric. (Piracicaba, Braz.), v.65, n.4, p.374-380, July/August 2008 
Table 2 - Traditional and saturated salt accelerated aging $\left(41^{\circ} \mathrm{C}\right.$ and $45^{\circ} \mathrm{C}$ for 48 and $\left.72 \mathrm{~h}\right)$ and controlled deterioration $(20 \%$, $22 \%$ and $24 \%$ seed moisture content) tests of six cauliflower seed lots, cv. Sharon, in two evaluation times.

\begin{tabular}{|c|c|c|c|c|c|c|c|c|c|c|c|c|}
\hline \multirow{3}{*}{ Evaluation } & \multirow{3}{*}{ Lots } & \multicolumn{4}{|c|}{$\begin{array}{c}\text { Traditional accelerated } \\
\text { aging }\end{array}$} & \multicolumn{4}{|c|}{$\begin{array}{l}\text { Accelerated aging with } \\
\text { saline solution }\end{array}$} & \multirow{2}{*}{\multicolumn{3}{|c|}{$\begin{array}{c}\text { Controlled deterioration } \\
\text { Moisture level (\%) }\end{array}$}} \\
\hline & & \multicolumn{2}{|c|}{$41^{\circ} \mathrm{C}$} & \multicolumn{2}{|c|}{$45^{\circ} \mathrm{C}$} & \multicolumn{2}{|c|}{$41^{\circ} \mathrm{C}$} & \multicolumn{2}{|c|}{$45^{\circ} \mathrm{C}$} & & & \\
\hline & & $48 \mathrm{~h}$ & $72 \mathrm{~h}$ & $48 \mathrm{~h}$ & $72 \mathrm{~h}$ & $48 \mathrm{~h}$ & $72 \mathrm{~h}$ & $48 \mathrm{~h}$ & $72 \mathrm{~h}$ & 20 & 22 & 24 \\
\hline & & $-\cdots$ & & 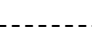 & & & o & & & & & - \\
\hline \multirow{7}{*}{$\begin{array}{l}1^{\text {st }} \\
(2003)\end{array}$} & 1 & $92 \mathrm{~b}$ & $83 \mathrm{c}$ & $26 \mathrm{c}$ & 0 & $92 \mathrm{a}$ & $90 \mathrm{a}$ & $92 \mathrm{a}$ & $73 \mathrm{ab}$ & $55 \mathrm{ab}$ & $46 \mathrm{ab}$ & $34 \mathrm{a}$ \\
\hline & 2 & $96 \mathrm{ab}$ & $92 a b c$ & $43 \mathrm{~b}$ & 0 & $97 \mathrm{a}$ & $91 \mathrm{a}$ & $93 \mathrm{a}$ & $69 \mathrm{~b}$ & $52 \mathrm{bc}$ & $52 \mathrm{ab}$ & $34 \mathrm{a}$ \\
\hline & 3 & 99 a & $94 \mathrm{ab}$ & $69 \mathrm{a}$ & 0 & $96 \mathrm{a}$ & $96 \mathrm{a}$ & $96 \mathrm{a}$ & $84 \mathrm{a}$ & $66 \mathrm{ab}$ & $60 \mathrm{a}$ & $49 \mathrm{a}$ \\
\hline & 4 & $94 \mathrm{~b}$ & $87 \mathrm{bc}$ & $25 \mathrm{c}$ & 0 & $94 \mathrm{a}$ & $93 \mathrm{a}$ & $90 \mathrm{a}$ & $67 \mathrm{~b}$ & $39 \mathrm{c}$ & $34 \mathrm{~b}$ & $34 \mathrm{a}$ \\
\hline & 5 & $98 \mathrm{ab}$ & $96 \mathrm{a}$ & $78 \mathrm{a}$ & 5 & $95 \mathrm{a}$ & $96 \mathrm{a}$ & $96 \mathrm{a}$ & $81 \mathrm{a}$ & $70 \mathrm{a}$ & $53 \mathrm{ab}$ & $49 \mathrm{a}$ \\
\hline & 6 & $94 \mathrm{~b}$ & $92 a b c$ & $74 \mathrm{a}$ & 0 & $94 \mathrm{a}$ & $89 a$ & $90 \mathrm{a}$ & $78 \mathrm{ab}$ & $55 \mathrm{ab}$ & $62 \mathrm{a}$ & $36 \mathrm{a}$ \\
\hline & CV (\%) & 5.1 & 5.2 & 9.6 & & 5.4 & 6.6 & 7.1 & 5.8 & 7.9 & 10.9 & 12.2 \\
\hline \multirow{7}{*}{$\begin{array}{l}2^{\text {nd }} \\
(2004)\end{array}$} & 1 & $92 \mathrm{ab}$ & $86 a b$ & $34 \mathrm{a}$ & --- & $94 \mathrm{a}$ & $89 \mathrm{ab}$ & $87 \mathrm{abc}$ & 89 a & $62 \mathrm{~b}$ & $47 \mathrm{~b}$ & $40 \mathrm{ab}$ \\
\hline & 2 & $93 \mathrm{ab}$ & $82 \mathrm{~b}$ & $0 \mathrm{c}$ & --- & $95 \mathrm{a}$ & $85 \mathrm{ab}$ & $76 \mathrm{c}$ & $73 \mathrm{~b}$ & $67 \mathrm{~b}$ & $47 \mathrm{~b}$ & $48 \mathrm{ab}$ \\
\hline & 3 & 96 a & $88 \mathrm{ab}$ & $2 c$ & --- & $97 \mathrm{a}$ & $95 \mathrm{ab}$ & $93 \mathrm{a}$ & $82 \mathrm{ab}$ & $78 \mathrm{ab}$ & $65 \mathrm{ab}$ & $53 \mathrm{a}$ \\
\hline & 4 & $83 \mathrm{~b}$ & $68 \mathrm{c}$ & $0 \mathrm{c}$ & --- & $89 a$ & $85 \mathrm{ab}$ & $83 a b c$ & $72 \mathrm{~b}$ & $42 \mathrm{c}$ & $28 \mathrm{c}$ & $26 \mathrm{~b}$ \\
\hline & 5 & $94 \mathrm{a}$ & $94 \mathrm{a}$ & $17 \mathrm{~b}$ & --- & $95 \mathrm{a}$ & $96 \mathrm{a}$ & $93 \mathrm{a}$ & $82 \mathrm{ab}$ & $84 \mathrm{a}$ & $77 \mathrm{a}$ & $62 \mathrm{a}$ \\
\hline & 6 & $88 \mathrm{ab}$ & $83 \mathrm{ab}$ & $10 \mathrm{~b}$ & --- & $93 \mathrm{a}$ & $82 \mathrm{~b}$ & $80 \mathrm{bc}$ & $74 \mathrm{~b}$ & $68 \mathrm{ab}$ & $53 \mathrm{~b}$ & $42 \mathrm{ab}$ \\
\hline & CV (\%) & 6.1 & 7.0 & 8.6 & -- & 6.8 & 7.8 & 7.2 & 5.9 & 8.9 & 10.3 & 14.6 \\
\hline
\end{tabular}

Lower case letters: lot comparison within each phase (Tukey test, $p \leq 0.05$ ).

dure, but showed relatively lower sensitivity to detect differences in seed vigor particularly when performed at $41^{\circ} \mathrm{C}$. The most consistent results of the saturated salt accelerated aging occurred for the combination $45^{\circ} \mathrm{C} / 72 \mathrm{~h}$, as the vigor of lots 2,4 and 6 was identified as the lowest while lots 3 and 5 were the best in performance.

In the electrical conductivity test, some seeds of the six lots exhibited protrusion of the primary root after 24-hour imbibition. For this reason, the data from this imbibition period were not submitted to statistical analysis and this procedure was not included in the second evaluation time. Differences in the leakage of seed lots that suggested different vigor levels was possible after four hours of imbibition in most combinations, as well as that verified in 12 hours (Table 3). However, few differences were significant at the first evaluation date. Higher conductivities and more differences were recorded at the second evaluation. However, lot 4 identified previously as possibly having lower vigor did not give a consistently higher conductivity, as could have been expected.

In general, the imbibition of 50 seeds in $75 \mathrm{~mL}$ for $4 \mathrm{~h}$ in the electrical conductivity test detected the lowest vigor of lots 4 and 6 and the best performance of lots 3 and 5. These results were similar to germination (second evaluation), accelerated aging and controlled deterioration. This information was also pro- vided by the potassium leachate test $(25$ seeds $/ 50 \mathrm{~mL} /$ $30 \mathrm{~min}$ ) as shown in Table 4. However, the other combinations for the potassium leachate test did not yield consistent results.

\section{DISCUSSION}

The adoption of high technology procedures have promoted a remarkable increase in the commercial production of many vegetable species, as a consequence of advances in research, in the demand of high quality seeds and in the improvement of quality control programs. The evaluation of seed physiological potential by the standard germination test has been considered incomplete as it does not estimate seed performance under moderately unfavorable or stressful field conditions. Consequently, seed vigor tests have attained increasing importance.

Jianhua \& McDonald (1997); Panobianco \& Marcos-Filho, (2001a); Martins et al. (2002); Rodo \& Marcos-Filho (2003); Torres \& Marcos-Filho (2003); Marcos-Filho et al. (2006) have documented the reliability of the saturated salt accelerated aging test to detect differences in the physiological potential of vegetable seeds. However, aging of cauliflower seeds at $41^{\circ} \mathrm{C}$ in this research did not show sensitiveness to identify seed vigor differences among seed lots, thus confirming the results reported by Paiva et al. (2005). 
Table 3 - Electrical conductivity test of six cauliflower seed lots, cv. Sharon, in two evaluation times.

\begin{tabular}{|c|c|c|c|c|c|c|c|c|c|}
\hline \multirow{3}{*}{ Evaluation } & \multirow{3}{*}{ Lots } & \multicolumn{4}{|c|}{4 hours } & \multicolumn{4}{|c|}{12 hours } \\
\hline & & \multicolumn{2}{|c|}{25 seeds } & \multicolumn{2}{|c|}{50 seeds } & \multicolumn{2}{|c|}{25 seeds } & \multicolumn{2}{|c|}{50 seeds } \\
\hline & & $25 \mathrm{~mL}$ & $50 \mathrm{~mL}$ & $50 \mathrm{~mL}$ & $75 \mathrm{~mL}$ & $25 \mathrm{~mL}$ & $50 \mathrm{~mL}$ & $50 \mathrm{~mL}$ & $75 \mathrm{~mL}$ \\
\hline & & - - & 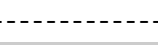 & 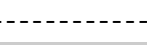 & - & $g \quad-\cdots-$ & & & - \\
\hline \multirow{6}{*}{$\begin{array}{l}1^{\text {st }} \\
(2003)\end{array}$} & 1 & $129.97 \mathrm{a}$ & $80.25 \mathrm{a}$ & $69.51 \mathrm{a}$ & $47.35 \mathrm{ab}$ & $194.53 \mathrm{a}$ & $122.03 \mathrm{a}$ & $100.30 \mathrm{a}$ & $70.23 \mathrm{ab}$ \\
\hline & 2 & $145.14 \mathrm{a}$ & $84.72 \mathrm{a}$ & $75.40 \mathrm{a}$ & $49.26 \mathrm{ab}$ & $197.68 \mathrm{a}$ & $123.21 \mathrm{a}$ & $102.69 \mathrm{a}$ & $69.20 \mathrm{ab}$ \\
\hline & 3 & $122.89 \mathrm{a}$ & $65.74 \mathrm{a}$ & $69.30 \mathrm{a}$ & $44.64 \mathrm{a}$ & $168.51 \mathrm{a}$ & $102.60 \mathrm{a}$ & $92.22 \mathrm{a}$ & $62.30 \mathrm{a}$ \\
\hline & 4 & $180.08 \mathrm{a}$ & $77.31 \mathrm{a}$ & $77.30 \mathrm{a}$ & $54.97 \mathrm{bc}$ & $232.25 \mathrm{a}$ & $121.80 \mathrm{a}$ & $115.67 \mathrm{ab}$ & $81.20 \mathrm{ab}$ \\
\hline & 5 & $111.60 \mathrm{a}$ & $66.04 \mathrm{a}$ & $67.40 \mathrm{a}$ & $44.92 \mathrm{a}$ & $159.91 \mathrm{a}$ & $96.86 \mathrm{a}$ & $93.67 \mathrm{a}$ & $64.38 \mathrm{ab}$ \\
\hline & 6 & $170.63 \mathrm{a}$ & $88.17 \mathrm{a}$ & $98.91 \mathrm{~b}$ & $61.07 \mathrm{c}$ & $235.66 \mathrm{a}$ & $138.82 \mathrm{a}$ & $139.06 \mathrm{~b}$ & $91.02 \mathrm{~b}$ \\
\hline $\mathrm{CV}(\%)$ & & 23.9 & 15.4 & 11.3 & 8.2 & 20.4 & 14.4 & 13.4 & 10.2 \\
\hline \multirow{6}{*}{$\begin{array}{l}2^{\text {nd }} \\
(2004)\end{array}$} & 1 & $177.24 \mathrm{ab}$ & $102.03 \mathrm{ab}$ & $72.05 \mathrm{a}$ & $54.95 \mathrm{a}$ & $251.09 \mathrm{ab}$ & $145.99 \mathrm{a}$ & $107.10 \mathrm{a}$ & $86.89 \mathrm{ab}$ \\
\hline & 2 & $183.43 \mathrm{~b}$ & $95.74 \mathrm{ab}$ & $98.34 \mathrm{c}$ & $55.27 \mathrm{ab}$ & $238.84 \mathrm{ab}$ & $123.13 \mathrm{a}$ & $134.82 \mathrm{bc}$ & $91.07 \mathrm{ab}$ \\
\hline & 3 & $146.32 \mathrm{ab}$ & $83.59 \mathrm{ab}$ & $68.36 \mathrm{a}$ & $53.31 \mathrm{ab}$ & $226.63 \mathrm{a}$ & $110.41 \mathrm{a}$ & $104.82 \mathrm{a}$ & $72.22 \mathrm{a}$ \\
\hline & 4 & $155.00 \mathrm{ab}$ & $96.74 \mathrm{ab}$ & $74.48 \mathrm{a}$ & $58.85 \mathrm{ab}$ & $263.61 \mathrm{ab}$ & $138.01 \mathrm{a}$ & $113.99 \mathrm{ab}$ & $91.09 \mathrm{ab}$ \\
\hline & 5 & $123.06 \mathrm{a}$ & $79.70 \mathrm{a}$ & $77.90 \mathrm{ab}$ & $51.66 \mathrm{a}$ & $209.07 \mathrm{a}$ & $110.29 \mathrm{a}$ & $112.76 \mathrm{ab}$ & $70.52 \mathrm{a}$ \\
\hline & 6 & $200.98 \mathrm{~b}$ & $102.79 \mathrm{~b}$ & $97.10 \mathrm{bc}$ & $66.82 \mathrm{~b}$ & $303.62 \mathrm{~b}$ & $123.98 \mathrm{a}$ & $141.73 \mathrm{c}$ & $105.77 \mathrm{~b}$ \\
\hline $\mathrm{CV}(\%)$ & & 15.9 & 10.8 & 10.8 & 11.4 & 11.7 & 17.2 & 9.3 & 15.0 \\
\hline
\end{tabular}

Lower case letters: lot comparison within each phase (Tukey test, $p \leq 0.05$ ).

Table 4 - Potassium leachate test of six cauliflower seed lots, cv. Sharon, in two evaluation times.

\begin{tabular}{|c|c|c|c|c|c|c|c|c|c|}
\hline \multirow{3}{*}{ Evaluation } & \multirow{3}{*}{ Lots } & \multicolumn{4}{|c|}{4 hours } & \multicolumn{4}{|c|}{12 hours } \\
\hline & & \multicolumn{2}{|c|}{25 seeds } & \multicolumn{2}{|c|}{50 seeds } & \multicolumn{2}{|c|}{25 seeds } & \multicolumn{2}{|c|}{50 seeds } \\
\hline & & $25 \mathrm{~mL}$ & $50 \mathrm{~mL}$ & $50 \mathrm{~mL}$ & $75 \mathrm{~mL}$ & $25 \mathrm{~mL}$ & $50 \mathrm{~mL}$ & $50 \mathrm{~mL}$ & $75 \mathrm{~mL}$ \\
\hline & & 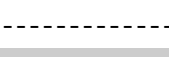 & 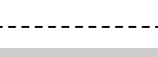 & -2 & -.... mg & N & 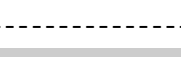 & -2 & - \\
\hline \multirow{6}{*}{$\begin{array}{l}1^{\text {st }} \\
(2003)\end{array}$} & 1 & $331.01 \mathrm{ab}$ & $376.51 \mathrm{ab}$ & $219.96 \mathrm{a}$ & $288.50 \mathrm{a}$ & $717.38 \mathrm{ab}$ & $854.81 \mathrm{ab}$ & $625.05 \mathrm{a}$ & $762.73 \mathrm{a}$ \\
\hline & 2 & $345.64 \mathrm{ab}$ & $406.52 \mathrm{ab}$ & $326.93 \mathrm{~b}$ & $337.65 \mathrm{a}$ & $771.50 \mathrm{ab}$ & $909.45 \mathrm{ab}$ & $793.10 \mathrm{ab}$ & $828.60 \mathrm{ab}$ \\
\hline & 3 & $302.11 \mathrm{a}$ & 326.86 a & $294.41 \mathrm{ab}$ & $294.29 \mathrm{a}$ & $694.86 \mathrm{a}$ & $715.44 \mathrm{a}$ & $698.33 \mathrm{a}$ & $742.55 \mathrm{a}$ \\
\hline & 4 & $322.43 \mathrm{ab}$ & $415.48 \mathrm{ab}$ & $283.51 \mathrm{ab}$ & $320.39 \mathrm{a}$ & $697.09 \mathrm{a}$ & $884.44 \mathrm{ab}$ & $695.67 \mathrm{a}$ & $761.36 \mathrm{a}$ \\
\hline & 5 & $349.70 \mathrm{ab}$ & $350.14 \mathrm{a}$ & $284.54 \mathrm{ab}$ & $333.55 \mathrm{a}$ & $762.81 \mathrm{ab}$ & $758.94 \mathrm{a}$ & 665.42 a & $740.98 \mathrm{a}$ \\
\hline & 6 & $433.23 \mathrm{~b}$ & $514.93 \mathrm{~b}$ & $458.18 \mathrm{c}$ & $477.48 \mathrm{~b}$ & $902.15 \mathrm{~b}$ & $1031.17 \mathrm{~b}$ & $980.83 \mathrm{~b}$ & $991.40 \mathrm{~b}$ \\
\hline $\mathrm{CV}(\%)$ & & 16.5 & 15.2 & 13.8 & 14.3 & 11.5 & 13.0 & 12.0 & 10.2 \\
\hline \multirow{6}{*}{$\begin{array}{l}2^{\text {nd }} \\
(2004)\end{array}$} & 1 & $338.1 .9 \mathrm{a}$ & $358.32 \mathrm{ab}$ & $241.51 \mathrm{a}$ & $348.35 \mathrm{a}$ & $694.20 \mathrm{a}$ & $792.86 \mathrm{ab}$ & $595.01 \mathrm{a}$ & $741.14 \mathrm{a}$ \\
\hline & 2 & $362.58 \mathrm{ab}$ & $401.15 \mathrm{~b}$ & $325.55 \mathrm{~b}$ & $405.69 \mathrm{ab}$ & $741.92 \mathrm{ab}$ & $888.70 \mathrm{~b}$ & $727.95 \mathrm{~b}$ & $796.17 \mathrm{ab}$ \\
\hline & 3 & $280.96 \mathrm{a}$ & 330.66 a & $248.03 \mathrm{a}$ & $359.44 \mathrm{a}$ & $613.27 \mathrm{a}$ & $793.51 \mathrm{ab}$ & $586.75 \mathrm{a}$ & $718.84 \mathrm{a}$ \\
\hline & 4 & $315.20 \mathrm{a}$ & $388.24 \mathrm{ab}$ & $272.19 \mathrm{ab}$ & $376.10 \mathrm{a}$ & $674.91 \mathrm{a}$ & $843.23 \mathrm{ab}$ & $683.09 \mathrm{ab}$ & $806.67 \mathrm{ab}$ \\
\hline & 5 & $290.19 \mathrm{a}$ & $325.98 \mathrm{a}$ & $251.02 \mathrm{a}$ & $319.91 \mathrm{a}$ & $637.59 \mathrm{a}$ & $682.39 \mathrm{a}$ & $604.89 \mathrm{a}$ & $712.90 \mathrm{a}$ \\
\hline & 6 & $456.80 \mathrm{~b}$ & $502.90 \mathrm{c}$ & $282.51 \mathrm{ab}$ & $479.60 \mathrm{~b}$ & $940.92 \mathrm{~b}$ & $986.74 \mathrm{~b}$ & $755.98 \mathrm{~b}$ & $929.03 \mathrm{~b}$ \\
\hline $\mathrm{CV}(\%)$ & & 13.5 & 8.0 & 11.7 & 11.0 & 12.5 & 11.0 & 8.3 & 8.0 \\
\hline
\end{tabular}

Lower case letters: lot comparison within each phase (Tukey test, $p \leq 0.05$ ).

The promising results reported here for the use of the saturated salt accelerated aging at $45^{\circ} \mathrm{C}$ for $72 \mathrm{~h}$ were similar to the findings of Fessel et al. (2005) to assess broccoli seed vigor.

The use of the controlled deterioration test identified differences between the cauliflower seed lots. This confirmed the observations of Matthews (1980);
Powell \& Matthews (1981); Panobianco \& MarcosFilho (2001a); Torres \& Marcos-Filho (2005) and Basak et al. (2006) for other vegetable seeds. The adjustment of seed water content to $20 \%$ or $22 \%$ produced the clearest separation between lots.

In this research, the electrical conductivity test separated seed lots into different vigor levels after a 
4h imbibition period as well as the potassium leachate test after 30 min imbibition. Some differences in sensitivity among vigor tests were observed with respect to the classification of the seed lots, but in general lots 4 and 6 were identified as having potentially the lowest performance. In addition the saturated salt accelerated aging test also suggested that lot 2 would have low vigor. This can be explained by the different principles on which each test is based, i.e., the electrical conductivity and potassium leachate tests evaluate indirectly the loss of cell membrane integrity through the quantification of ion leakage, but the accelerated aging and controlled deterioration focus on seed performance after the exposure to temperature and relative humidity or seed moisture content stressing conditions. As a consequence, the use of a combination of two or three tests is recommended to provide a reliable estimation of the physiological potential of cauliflower seed lots.

Based on the results of this research, the tests of traditional accelerated aging $\left(41^{\circ} \mathrm{C}\right.$ for $\left.48 \mathrm{~h}\right)$, the saturated salt accelerated aging $\left(45^{\circ} \mathrm{C}\right.$ for $\left.72 \mathrm{~h}\right)$, and the controlled deterioration (seed moisture level adjusted to $20 \%$ or $22 \%$, at $45^{\circ} \mathrm{C}$, for $24 \mathrm{~h}$ ) consistently identified differences between cauliflower seed lots that may reflect their physiological potential. Likewise, the imbibition of 50 seeds in $75 \mathrm{~mL}$ water during $4 \mathrm{~h}$ for the electrical conductivity test or the combination 25 seeds/ $50 \mathrm{~mL}$ water $/ 30$ minutes imbibition at $25^{\circ} \mathrm{C}$ for the potassium leachate test allow the separation of lots into different vigor levels.

The vigor tests performed here ranked the potential performance of seed lots, but seedling emergence failed to detect differences in seed physiological potential. Since all seed lots presented high germination rate, above market standards, the favorable environmental conditions were not sufficient to result in reduced seedling emergence percentage when planted under greenhouse conditions.

It is difficult to establish a direct relationship between seed germination or vigor and stand establishment since seedling emergence is a consequence of physiological potential and environmental conditions of the seedbed (Egli \& TeKrony, 1995). As a result, measures of seed vigor usually provide better estimates mainly when seeds are exposed to unfavorable conditions. In addition, large differences in seed vigor are necessary to show a positive relationship with seedling field emergence (Burris, 1976). This means that acceptable stands may be obtained in spite of differences in vigor levels. This probably occurs under favorable environmental conditions in the field when the stands of both high and low vigor seed lots are usually similar in relation to their germination percentages (Venter, 2000).
In conclusion, results here presented provide useful information regarding the identification of alternatives to assess cauliflower seed vigor in the laboratory. The tests indicated here should be considered as promising for successful seed quality control programs. However, the ability of some of these tests to define seed vigor of cauliflower, as reflected in emergence and/or storage potential, needs additional studies including less favorable conditions at sowing time in the field or during storage.

\section{ACKNOWLEDGEMENTS}

To FAPESP and CNPq for financial support, and to Sakata Seed Sudamerica Ltda., for providing the cauliflower seed lots.

\section{REFERENCES}

BASAK, O.; DEMIR, I.; MAVI, K.; MATTHEWS, S. Controlled deterioration test for predicting seedling emergence and longevity of pepper (Capsicum annuиm L.) seed lots. Seed Science and Technology, v.34, p.701-712, 2006.

BRASIL. Ministério da Agricultura e Reforma Agrária. Regras para análise de sementes. Brasília: SNDA/DNDV/CLAV, 1992. 365p.

BURRIS, J.S. Seed/seedling vigor and field performance. Journal of Seed Technology, v.1, p.58-74, 1976.

CUSTÓDIO, C.C.; MARCOS-FILHO, J. Potassium leachate test for the evaluation of soybean seed physiological quality. Seed Science and Technology, v.25, p.549-564, 1997.

DIAS, D.C.F.S.; VIEIRA, A.N.; BHERING, M.C. Condutividade elétrica e lixiviação de potássio para avaliação do vigor de sementes de hortaliças: feijão, vagem e quiabo. Revista Brasileira de Sementes, v.20, p.408-413, 1998.

EGLI, D.B.; TeKRONY, D.M. Soybean seed germination, vigor and field emergence. Seed Science and Technology, v.23, p.595-607, 1995.

FESSEL, S.A.; SILVA, L.J.R.; GALLI, J.A.; SADER, R. Uso de solução salina $(\mathrm{NaCl})$ no teste de envelhecimento acelerado em sementes de brócolis (Brassica oleracea L. var. itálica Plenk). Científica, v.33, p.27-34, 2005.

JIANHUA, Z.; McDONALD, M.B. The saturated salt accelerated aging test for small seeded crops. Seed Science and Technology, v.25, p.123-131, 1997.

MARCOS-FILHO, J. Teste de envelhecimento acelerado. In: KRZYZANOWSKI, F.C.; VIEIRA, R.D.; FRANÇA NETO, J.B. (Ed.) Vigor de sementes: conceitos e testes. Londrina: ABRATES, 1999. cap.3, p.1-24.

MARCOS-FILHO, J.; BENNETT, M.A.; McDONALD, M.B.; EVANS, A.F.; GRASSBAUGH, E.M. Assessment of melon seed vigour by an automated computer imaging system compared to traditional procedures. Seed Science and Technology, v.34, p.485-497, 2006.

MARTINS, C.C.; MARTINELLI-SENEME, A.; CASTRO, M.M.; NAKAGAWA, J.; CAVARIANI, C. Comparação entre métodos para a avaliação do vigor de lotes de sementes de couve-brócolos (Brasica oleracea L. var. italica Plenk). Revista Brasileira de Sementes, v.24, p.96-101, 2002.

MATTHEWS, S. Controlled deterioration: a new vigour test for crop seeds. In: HEBBLETHWAITE, P.D. Seed production. London: Butterworths, 1980. p.647-660.

OLIVEIRA, S.R.S.; NOVEMBRE, A.D.L.C. Teste de condutividade elétrica para as sementes de pimentão. Revista Brasileira de Sementes, v.27, p.31-36, 2005. 
PAIVA, A.S.; LOPES, M.M.; TESSER, S.M.; PANOBIANCO, M.; VIEIRA, R.D. Avaliação do potencial fisiológico de sementes de couve-flor. Científica, v.33, p.103-105, 2005.

PANOBIANCO, M.; MARCOS-FILHO, J. Envelhecimento acelerado e deterioração controlada em sementes de tomate. Scientia Agricola, v.58, p.525-531, 2001a.

PANOBIANCO, M.; MARCOS-FILHO, J. Evaluation of the physiological potential of tomato seeds by germination and vigor tests. Seed Technology, v.23, p.151-161, 2001b.

POWELL, A.A. The controlled deterioration test. In: VENTER, H.A. van der (Ed.) Seed vigour testing seminar. Copenhagen: International Seed Testing Association, 1995. p.73-87.

POWELL, A.A.; MATTHEWS, S. Evaluation of controlled deterioration, a new vigour test for small seed vegetables. Seed Science and Technology, v.9, p.633-640, 1981.

RODO, A.B.; MARCOS-FILHO, J. Accelerated aging and controlled deterioration for the determination of the physiological potential of onion seeds. Scientia Agricola, v. 60, p.465-469, 2003.
ROSSETTO, C.A.V.; FERNANDEZ, E.M.; MARCOS-FILHO, J. Metodologias de ajuste do grau de umidade e comportamento de sementes de soja no teste de envelhecimento acelerado. Revista Brasileira de Sementes, v.17, p.171-178, 1995.

TeKRONY, D.M. Accelerated aging In: VENTER, H.A. van der (Ed.) Seed vigour testing seminar. Copenhagen: International Seed Testing Association, 1995. p.53-72.

TORRES, S.B.; MARCOS-FILHO, J. Physiological potential evaluation in melon seeds (Cucumis melo L.). Seed Science and Technology, v.33, p.341-350, 2005.

TORRES, S.B.; MARCOS-FILHO, J. Accelerated aging of melon seeds. Scientia Agrícola, v.60, p.77-82, 2003

VENTER, H.A. van der. What is seed vigor? ISTA News Bulletin, n. 121, p. $12-13,2000$.

Received May 04, 2007

Accepted January 07, 2008 